ARTICLE

\title{
Giant field-like torque by the out-of-plane magnetic spin Hall effect in a topological antiferromagnet
}

\author{
Kouta Kondou (10) 1,2凶 , Hua Chen (10 ${ }^{3,4}$, Takahiro Tomita (1) 2,5, Muhammad Ikhlas ${ }^{2,5}$, Tomoya Higo ${ }^{2,6}$, \\ Allan H. MacDonald (10 7 , Satoru Nakatsuji ${ }^{2,5,6,8,9}$ \& YoshiChika Otani (1D) 1,2,5,8凶
}

Spin-orbit torques (SOT) enable efficient electrical control of the magnetic state of ferromagnets, ferrimagnets and antiferromagnets. However, the conventional SOT has severe limitation that only in-plane spins accumulate near the surface, whether interpreted as a spin Hall effect (SHE) or as an Edelstein effect. Such a SOT is not suitable for controlling perpendicular magnetization, which would be more beneficial for realizing low-powerconsumption memory devices. Here we report the observation of a giant magnetic-fieldlike SOT in a topological antiferromagnet $\mathrm{Mn}_{3} \mathrm{Sn}$, whose direction and size can be tuned by changing the order parameter direction of the antiferromagnet. To understand the magnetic SHE (MSHE)- and the conventional SHE-induced SOTs on an equal footing, we formulate them as interface spin-electric-field responses and analyzed using a macroscopic symmetry analysis and a complementary microscopic quantum kinetic theory. In this framework, the large out-of-plane spin accumulation due to the MSHE has an inter-band origin and is likely to be caused by the large momentum-dependent spin splitting in $\mathrm{Mn}_{3} \mathrm{Sn}$. Our work demonstrates the unique potential of antiferromagnetic Weyl semimetals in overcoming the limitations of conventional SOTs and in realizing low-power spintronics devices with new functionalities.

\footnotetext{
${ }^{1}$ RIKEN, Center for Emergent Matter Science (CEMS), Saitama 351-0198, Japan. ${ }^{2}$ CREST, Japan Science and Technology Agency (JST), Kawaguchi, Saitama 332-0012, Japan. ${ }^{3}$ Department of Physics, Colorado State University, Fort Collins, CO, USA. ${ }^{4}$ School of Advanced Materials Discovery, Colorado State University, Fort Collins, CO, USA. ${ }^{5}$ Institute for Solid State Physics, The University of Tokyo, Kashiwa, Chiba 277-8581, Japan. ${ }^{6}$ Department of Physics, University of Tokyo, Hongo, Bunkyo-ku, Tokyo 113-0033, Japan. ${ }^{7}$ Department of Physics, University of Texas at Austin, Austin, TX, USA. ${ }^{8}$ Trans-scale Quantum Science Institute, University of Tokyo, Tokyo, Japan. ${ }^{9}$ Institute for Quantum Matter and Department of Physics and Astronomy, Johns Hopkins University, Baltimore, MD 21218, USA.凶email: kkondou@riken.jp; yotani@issp.u-tokyo.ac.jp
} 
$\mathrm{n}$ the conventional nonmagnetic materials with high crystalline symmetry used in SHE-related experiments, currentinduced spin accumulation has its polarization parallel to the surface and perpendicular to the current direction ${ }^{1-6}$. Thus, the SOTs generated by the accumulated spins can only generate slow in-plane magnetization switching or precessional motion of outof-plane magnetization ${ }^{7}$. Therefore, efficient switching in magnetic devices with perpendicular magnetic anisotropy can be achieved only by applying an undesirable bias magnetic field ${ }^{8-10}$ or an effective magnetic field ${ }^{11-13}$. It is nevertheless imperative to have directional control of the current-generated spin accumulation and its resulting SOTs to realize low-power spintronic devices utilizing magnetic thin films with perpendicular magnetic anisotropy. With this motivation, recent experimental and theoretical studies have demonstrated the generation of out-of-plane components in the spin accumulation by using the anomalous Hall effect (AHE) of the ferromagnet ${ }^{14,15}$, antiferromagnets ${ }^{16,17}$, ferromagnet/nonmagnet metal interface effects ${ }^{18}$, and lowsymmetry materials such as transition-metal dichalcogenides (TMDs) ${ }^{19,20}$.

The antiferromagnetic Weyl semimetals (Weyl antiferromagnets) with chemical formula $\mathrm{Mn}_{3} X(X=\mathrm{Sn}, \mathrm{Ge})$ exhibit exotic physical properties such as the anomalous Hall effect ${ }^{21}$, the anomalous Nernst effect ${ }^{22}$, the magneto-optical Kerr effect ${ }^{23}$, and omnidirectional read-out ${ }^{24}$, despite having nearly compensated total spin magnetization. These properties are partly related to the large momentum-space Berry curvatures of their low-energy Weyl quasi-particles ${ }^{25,26}$. Remarkably, the magnetic states of these materials can be controlled by a small external magnetic field ${ }^{21}$ or a spin-orbit torque ${ }^{27}$, as if they were ferromagnets. It is thus anticipated that the topological Weyl antiferromagnet can offer new functionalities for spintronics. In fact, a novel magnetic spin Hall effect (MSHE) has been discovered in $\mathrm{Mn}_{3} \mathrm{Sn}$, in which the spin-polarization direction of current-induced spin accumulation changes its sign upon flipping the chiral antiferromagnetic order of $\mathrm{Mn}_{3} \mathrm{Sn}^{28}$. Interestingly, the theoretical calculations hinted at a sizeable out-of-plane component of the current-induced spin density due to the MSHE i.e., out-of-plane MSHE. Very recently, in the non-collinear antiferromagnet $\mathrm{Mn}_{3} \mathrm{GaN}^{16}$ and the collinear antiferromagnet $\mathrm{Mn}_{2} \mathrm{Au}^{17}$, SOTs by out-of-plane spin component have been observed by means of temperature or strain-induced magnetic order control. However no experimental confirmation in the Weyl antiferromagnet $\mathrm{Mn}_{3} X$ has been made to date.

Here we report the first experimental deomstration of out-ofplane MSHE and the associated giant field-like torque. Namely, its sign can be controlled by the direction of the staggered moments of the antiferromagnetic Weyl semimetal $\mathrm{Mn}_{3} \mathrm{Sn}$. Conceptually, SOTs at the interface between two magnetic materials require a new classification scheme compared to the standard field-like torque (FLT) vs. anti-damping spin-transfer torque (STT) classification applied at a nonmagnetic-metal/ferromagnet interface, especially when the two magnetic order parameters can be separately manipulated ${ }^{29}$. Therefore, here we take a symmetry-based approach and construct a microscopic theory based on the interface spin to electric-field response approach adopted in ref. ${ }^{28}$, without relying on spin currents and use it to separate STT and FLTs due to SHE and MSHEs.

\section{Results}

Detection of effective out-of-plane magnetic field due to MSHE. To investigate SOTs caused by the MSHE, the ST-FMR technique is applied to single-crystal $\mathrm{Mn}_{3} \mathrm{Sn} / \mathrm{Ni}$-Fe bilayers. STFMR is a powerful technique to evaluate current-induced SOTs in bilayer structures involving paramagnetic heavy metals ${ }^{30}$ or topological insulators ${ }^{31,32}$. A $\mathrm{Mn}_{3} \mathrm{Sn}$ rectangular strip with dimensions $10 \times 0.24 \times 55 \mu^{3}$ was cut out of a single crystal using a focused ion-beam technique, followed by e-beam deposition of a $20-\mathrm{nm}$ thick Ni-Fe layer on top of the strip surface.

Firstly, to verify the existence of an out-of-plane component of spin accumulation as predicted by theory ${ }^{28}$, we examine whether the spin accumulation can act as an effective out-of-plane magnetic field $H_{\text {eff }}^{z}$ by measuring a shift of the resonance field $H_{\mathrm{r}}$ in the ST-FMR spectrum under the application of dc current. Fig. $1 \mathrm{a}$ and $\mathrm{b}$ respectively show the measurement circuit and a typical ST-FMR spectrum. We can see a small shift, which may include not only a contribution from $H_{\text {eff }}^{z}$ but also that of the current induced in-plane magnetic field generated by the spinaccumulation due to the conventional SHE. We therefore excluded the influence of the current induced in-plane real and effective magnetic fields by focusing on the difference $\Delta H_{\mathrm{r}}$ between shifts measured for out-of-plane magnetic fields at polar angles $\theta=45^{\circ}$ and $135^{\circ}$ with fixed azimuthal angle $\varphi$. Fig. 1e shows $\Delta H_{\mathrm{r}}$ as a function of $I_{\mathrm{dc}}$ at $\varphi=-45^{\circ}$. The inset shows the spin structure in $\mathrm{Mn}_{3} \mathrm{Sn}$ for $\varphi=-45^{\circ}$. A positive slope in the variation of $\Delta H_{\mathrm{r}}$ with $I_{\mathrm{dc}}$ indicates that the $H_{\mathrm{eff}}^{z}$ is along the $+\mathrm{z}$ direction, as illustrated in Fig. 1c. On the other hand, the variation of $\Delta H_{\mathrm{r}}$ with $I_{\mathrm{dc}}$ for $\varphi=+135^{\circ}$ clearly shows a sign change of the slope that we associate with the reversed spin structure of $\mathrm{Mn}_{3} \mathrm{Sn}$. This observation demonstrates directional switching of the out-of-plane $\mathrm{MSHE}^{28}$.

Angular dependence of in-plane torque $\tau^{\varphi}$ and the out-of-plane torque $\tau^{\mathbf{z}}$. Notably, the above results demonstrate that the MSHE in $\mathrm{Mn}_{3} \mathrm{Sn}$ exerts a SOT on the adjacent ferromagnetic layer that can be tuned by changing the orientation of the magnetic octupoles. Therefore we have next studied the direction and magnitude of the SOT that depends on the magnetic octuopole orientation in $\mathrm{Mn}_{3} \mathrm{Sn}$ by measuring the in-plane azimuthal angle $\varphi$ dependence of ST-FMR spectrum in a $\mathrm{Mn}_{3} \mathrm{Sn} / \mathrm{Ni}-\mathrm{Fe}$ bilayer. Fig. 2a illustrates the measurement circuit together with a schematic explanation of the in-plane torque $\tau^{\varphi}$ and the out-of-plane torque $\tau^{\mathrm{Z}}$ exerted on the Ni-Fe magnetization in the $\mathrm{Mn}_{3} \mathrm{Sn} / \mathrm{Ni}-\mathrm{Fe}$ bilayer. When an rf current is applied to the bilayer stripe, the induced rf Oersted field $H_{\mathrm{Oe}}^{\mathrm{rf}}$ excites FMR in the top Ni-Fe layer under the application of external field $H_{\mathrm{ex}}$. The spin accumulation simultaneously appears at the $\mathrm{Mn}_{3} \mathrm{Sn} / \mathrm{Ni}-\mathrm{Fe}$ interface, which exerts SOTs linear in the rf current on the Ni-Fe magnetization.

Fig. $2 \mathrm{~b}$ shows a typical ST-FMR spectrum of a $\mathrm{Mn}_{3} \mathrm{Sn} / \mathrm{Ni}-\mathrm{Fe}$ bilayer. All measurements were carried out at room temperature. The input radio frequency and power are $13 \mathrm{GHz}$ and $7 \mathrm{~mW}$, respectively. The ST-FMR spectrum generally contains a symmetric component $V_{\mathrm{S}}=-\frac{1}{4} \frac{d R}{d \varphi} \frac{\gamma I_{\mathrm{rf}} \sin \varphi}{\delta 2 \pi(d f / d H)_{H_{\mathrm{ex}}=H_{\mathrm{r}}}} S_{\frac{\delta}{\delta^{2}+\left(H_{\mathrm{ex}}-H_{\mathrm{r}}\right)^{2}}}$ and an asymmetric component, $V_{\mathrm{A}}=-\frac{1}{4} \frac{d R}{d \varphi} \frac{\gamma I_{\mathrm{rf}} \sin \varphi}{\delta 2 \pi(d f / d H)_{H_{\mathrm{ex}}=H_{\mathrm{r}}}} A \frac{\left(H_{\mathrm{ex}}-H_{\mathrm{r}}\right)}{\delta^{2}+\left(H_{\mathrm{ex}}-H_{\mathrm{r}}\right)^{2}}$. Here $\mathrm{dR} / \mathrm{d} \varphi, \gamma, I_{\mathrm{rf}}, \varphi, \delta, S$ and $A$ are respectively the sample resistance change due to precessional motion, the gyromagnetic ratio, the input rf current, the in-plane magnetic field angle, the half-width at half maximum of the ST-FMR spectrum and the coefficients of the symmetric and asymmetric components ${ }^{30,33,34}$. The torques $\left(\tau^{\varphi}\right.$ and $\tau^{Z}$ ) per moment exerted on the Ni-Fe layer measured in Tesla can be obtained from the values of $V_{\mathrm{S}}$ and $V_{\mathrm{A}}$ through $\tau^{\varphi(z)}=-8 \pi V_{S(A)} \delta\left(\frac{d f}{d H}\right) /\left\{\left(\frac{d R}{d \varphi}\right) \gamma I_{r f}\right\}$. Note that $V_{\mathrm{S}}$ and $V_{\mathrm{A}}$ respectively correspond to $\tau^{\varphi}$ and $\tau^{\mathrm{Z}}$ regardless of the latter's microscopic mechanism. In the case of SOTs due to a conventional SHE in the diffusive transport regime, the bulk spin current in the nonmagnetic metal leads to interface spin accumulation polarized along the $y$-axis even in the absence of the 
a

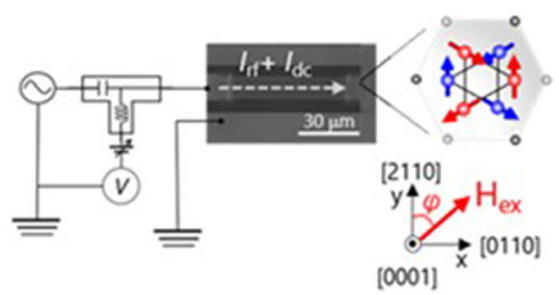

b

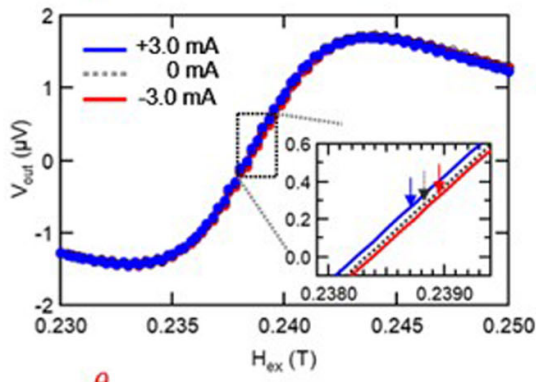

d

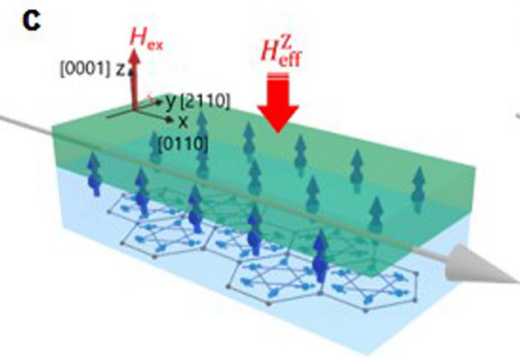

e

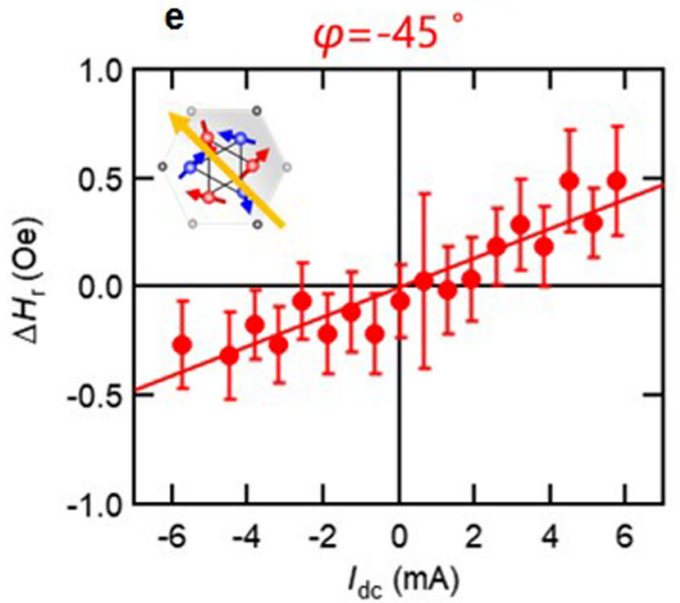

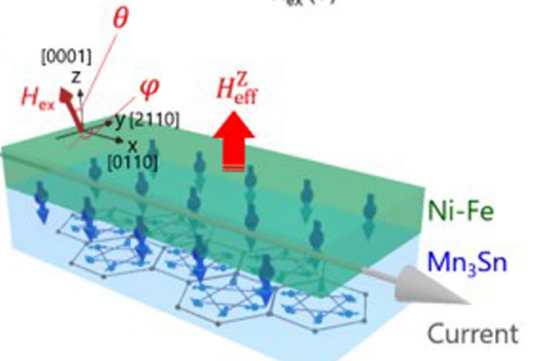

f

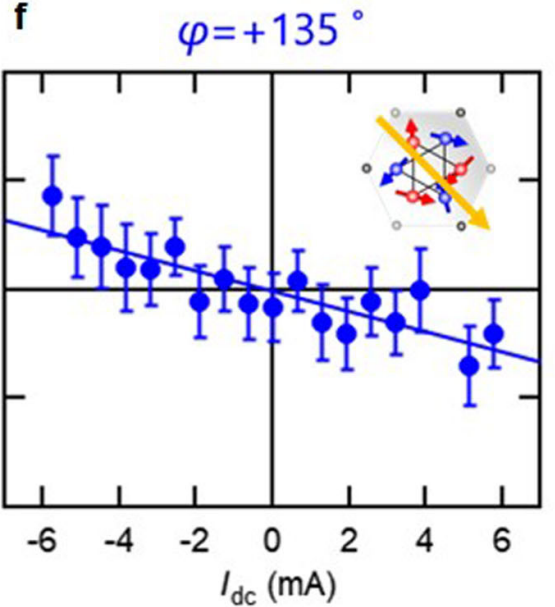

Fig. 1 Detection of the effective field depending on the magnetic octupole orientation in $\mathbf{M n}_{\mathbf{3}} \mathbf{S n}$. a Measurement circuit, $\mathbf{b}$ ST-FMR resonance spectrum in $\mathrm{Mn}_{3} \mathrm{Sn} / \mathrm{Ni}$-Fe bilayer. c, $\mathbf{d}$ Schematic image of an out-of-plane component of spin accumulation at $\mathrm{Mn}_{3} \mathrm{Sn} / \mathrm{Ni}$-Fe interface. e, $\mathbf{f}$ Resonance field shift by dc current $\left(\mathbf{e} \Delta H_{\mathrm{r}}=H_{\mathrm{r}}\left(\varphi=-45^{\circ}, \theta=45^{\circ}\right)-H_{\mathrm{r}}\left(\varphi=-45^{\circ}, \theta=135^{\circ}\right)\right.$, f $\left.\Delta H_{\mathrm{r}}=H_{\mathrm{r}}\left(\varphi=135^{\circ}, \theta=45^{\circ}\right)-H_{\mathrm{r}}\left(\varphi=135^{\circ}, \theta=135^{\circ}\right)\right)$ Error bars represent the standard deviation of five replicate measurements. Input radio frequency and power are $13 \mathrm{GHz}$ and $7 \mathrm{~mW}$.

ferromagnetic layer, which gives rise to $\tau^{z} \propto\left(\mathbf{m} \times \delta s_{y} \hat{\mathbf{e}}_{y}\right) \cdot \hat{\mathbf{e}}_{z}$, where $\mathbf{m}$ and $\delta$ s are the magnetization directions of the ferromagnet and the spin accumulation, respectively. The torque $\tau^{\mathrm{z}}$, in this case, is a purely FLT since it is odd under $\mathbf{m} \rightarrow-\mathbf{m}$. The spin current from both the bulk of the nonmagnetic layer and the diffusion of the accumulated spins into the ferromagnetic layer then leads to the STT $\boldsymbol{\tau}^{\varphi} \propto \mathbf{m} \times\left(\delta s_{y} \hat{\mathbf{e}}_{y} \times \mathbf{m}\right)$. The torque $\boldsymbol{\tau}^{\varphi}$ is therefore purely a STT since it is even under the reversal of $\mathbf{m}$. In our discussion below, the scalar $\tau^{\varphi}$ is the in-plane torque projected to the direction of $\hat{\mathbf{e}}_{z} \times \mathbf{m}$ and therefore has opposite parity to $\boldsymbol{\tau}^{\varphi}$ under $\mathbf{m}$ reversal. However, in more general cases with lower symmetry, $\tau^{\mathrm{Z}}$ and $\tau^{\varphi}$ are neither purely even nor odd under $\mathbf{m} \rightarrow-\mathbf{m}$. For example, a $\delta s_{z}$ spin accumulation can lead to a field-like $\tau^{\varphi}$. A further complication in the present system comes from the nontrivial dependence of the accumulated spin on the order parameter direction of $\mathrm{Mn}_{3} \mathrm{Sn}$.

To separate the different contributions to $\tau^{\mathrm{Z}}$ and $\tau^{\varphi}$ in the present system, we have measured their dependence on the in-plane magnetic field direction i.e., the azimuthal angle $\varphi$. The plane of the external magnetic field $H_{\mathrm{ex}}$ rotation corresponds to the kagome plane in
$\mathrm{Mn}_{3} \mathrm{Sn}$. Fig. $2 \mathrm{c}$ shows the schematic illustration of spin structures together with the octupole orientation in $\mathrm{Mn}_{3} \mathrm{Sn}$, aligned along an external magnetic field that is larger than the switching field of $\mathrm{Mn}_{3} \mathrm{Sn}^{28}$. Fig. $2 \mathrm{~d}$ and e show the azimuthal angle $\varphi$ dependence of the magnitudes of $V_{\mathrm{S}}$ and $V_{\mathrm{A}}$ in the $\mathrm{Mn}_{3} \mathrm{Sn} / \mathrm{Ni}-\mathrm{Fe}$ bilayer, from which one can obtain $\tau^{\varphi}$ and $\tau^{z}$ as shown in Fig. 2 f and g.

The angular dependence of $\tau^{\varphi}$ in the $\mathrm{Mn}_{3} \mathrm{Sn} / \mathrm{Ni}$-Fe bilayer (Fig. 2f) is entirely different from that of STT due to conventional SHE (Supplementary Fig. 1). The conventional SHE induced STT signal exhibits a simple $\sin \varphi$ dependence due to the projection of $\boldsymbol{\tau}^{\varphi} \propto \mathbf{m} \times\left(\delta s_{y} \hat{\mathbf{e}}_{y} \times \mathbf{m}\right)$ onto $\hat{\mathbf{e}}_{z} \times \mathbf{m}$. Such a $\tau^{\varphi}$ reaches a maximum when the magnetization of Ni-Fe is (anti) parallel to the rf charge current, i.e. $\varphi= \pm 90^{\circ}$ (Supplementary Fig. 1). However, the $\tau^{\varphi}$ in $\mathrm{Mn}_{3} \mathrm{Sn} / \mathrm{Ni}-\mathrm{Fe}$ bilayer has local maxima and minima at around $\varphi=$ $\pm 45^{\circ}$ and $\pm 135^{\circ}$. The angular variations of the direction and amplitude of the experimentally determined $\tau^{\varphi}$ are illustrated in the upper row of Fig. 2c.

Analysis based on interface spin-electric-field responses. The unusual angular dependence in the present system calls for a 


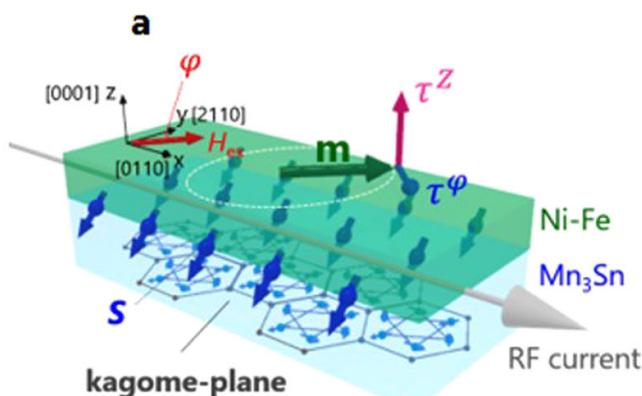

C
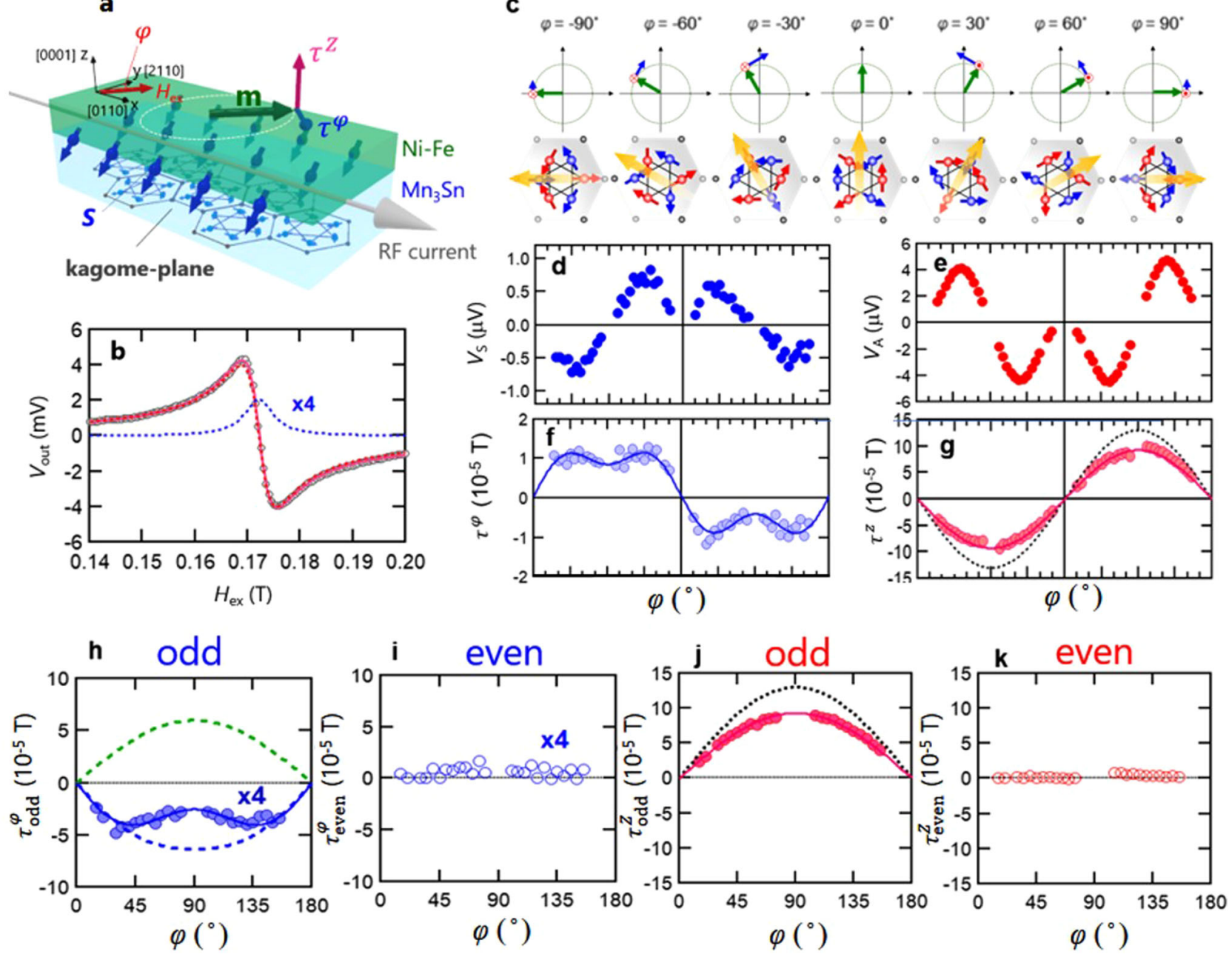

Fig. 2 Spin-torque FMR measurements in a $\mathbf{M n}_{3} \mathbf{S n} / \mathbf{N i}$-Fe bilayer. a Measurement setup for spin-torque FMR measurement (ST-FMR). The red and blue arrows on the respective top and bottom surface of the $\mathrm{Mn}_{3} \mathrm{Sn}$ stack indicates spin accumulation. $\mathbf{b} \mathrm{ST}$-FMR spectrum. c (Upper) Magnetization of $\mathrm{Ni}$-Fe (green arrows) and detected in-plane torque $\tau^{\varphi}$ (blue arrows) and out-of-plane torque $\tau^{Z}$ (red) in $\mathrm{Mn}_{3} \mathrm{Sn} / \mathrm{Ni}$-Fe bilayer system; (Lower) Spin structure of $\mathrm{Mn}_{3} \mathrm{Sn}$ and cluster magnetic octupole (orange arrows). d, e In-plane magnetic field angle dependence of symmetric voltage $V_{\mathrm{S}}$ and Antisymmetric voltages $V_{\mathrm{A}} . \mathbf{f}, \mathbf{g}$ In-plane torque $\tau^{\varphi}$ and out-of-plane torque $\tau^{Z}$. $\mathbf{h}, \mathbf{i}$ odd- and even-component of $\tau^{\varphi}$ under reversal of the ferromagnetic magnetization $\mathbf{m}$. Green (Black) dashed line corresponds to $\tau^{\varphi}$ due to conventional SHE (MSHE). $\mathbf{j}$, $\mathbf{k}$ Odd- and even-component of $\tau^{z}$. Black dashed line corresponds to $\tau^{z}$ due to Oersted field.

revisit of the conventional spin-current picture of SOT in bilayer structures. In ref. ${ }^{28}$, we have defined the (M)SHE as the boundary spin to electric-field response, avoiding conceptual difficulties that arise in defining both spin currents in strongly spin-orbit-coupled systems and spin-currents that flow in the direction perpendicular to a $2 \mathrm{D}$ system. In the case of the SOT, one can still define $\tau$ as the torque due to the current- or electricfield-induced spin density $\delta s^{28}$, since it is exchange-coupled to the magnetization $\mathbf{m}$ of the ferromagnetic layer. In this description, the FLT and STT in conventional ferromagnetic/nonmagneticmetal bilayers correspond respectively to $\delta$ s contributions that are even and odd under $\mathbf{m} \rightarrow-\mathbf{m}^{35-37}$. When the nonmagnetic metal is replaced by a magnetic one, such as $\mathrm{Mn}_{3} \mathrm{Sn}, \delta$ s additionally depends on its magnetic order parameter denoted as $\mathbf{n}$. The SHE and the MSHE can be distinguished by their parity under $\mathbf{n} \rightarrow-\mathbf{n}$. We can therefore describe STTs and FLTs due to the SHE and the MSHE by using a single spin-electric-field response function, and examining its dependence on the separate reversal of $\mathbf{m}$ and $\mathbf{n}$ (Table S1). For example, the STT (FLT) due to the MSHE results is the part of $\delta \mathbf{s}$ that is odd (even) in $\mathbf{m}$ and odd in $\mathbf{n}$.
Based on this new picture, we performed symmetry analysis of the spin-electric-field response function $\chi$, defined through $\delta s=\chi$ E with $\mathbf{E}$ being the electric field, at the $\mathrm{Ni}-\mathrm{Fe} / \mathrm{Mn}_{3} \mathrm{Sn}$ interface, which has a $C_{3 v}$ symmetry determined by $\mathrm{Mn}_{3} \mathrm{Sn}$ since the $\mathrm{Ni}-\mathrm{Fe}$ layer is polycrystalline. By truncating the Fourier expansion in order parameter direction at $2^{\text {nd }}$ order, and taking into account the opposite rotation of the weak magnetization to the sublattice moments of $\mathrm{Mn}_{3} \mathrm{Sn}$, we obtain the following angular dependence of $\delta s_{z}$ corresponding to the FLT component of the MSHE:

$$
\delta s_{z}=\left(b_{2}+d_{5} \sin ^{2} \varphi+d_{6} \cos ^{2} \varphi\right) \sin \varphi
$$

where $b_{2}, d_{5}$, and $d_{6}$ are system-dependent parameters. $\tau_{\text {odd }}^{\varphi}$ has the same angular dependence since $\tau_{\text {odd }}^{\varphi} \propto\left(\boldsymbol{m} \times \delta s_{z} \hat{\mathbf{e}}_{z}\right) \cdot\left(\boldsymbol{m} \times \hat{\mathbf{e}}_{z}\right) \propto \delta s_{z}$. However, one should note that the $\delta s_{z}$ corresponding to the STT component of the SHE also has the same form of angular dependence as in Eq. (1), and cannot be separated from the latter in this symmetry approach. On the other hand, the $\delta s_{z}$ contributing to $\tau_{\text {even }}^{\varphi}$ has the angular dependence of $c_{4} \cos 2 \varphi+c_{8}$, where the two terms are respectively due to the FLT of SHE and the STT of MSHE, provided 
that the truncation of the order parameter directions at 2 nd order is approximately valid.

To complement the symmetry analysis with a microscopic formalism for calculating the STT and FLT of (M)SHE separately, we adopt the quantum kinetic theory with the constant relaxation time $(\tau)$ approximation as in ref. ${ }^{28}$. In this approximation the $\mathrm{O}\left(\tau^{0}\right)$ contribution to $\chi$ includes the STT component of the SHE and the FLT component of the MSHE; whereas the $\mathrm{O}\left(\tau^{1}\right)$ contribution includes the FLT component of the SHE and the STT of the MSHE (Table S2). The SHE and MSHE contributions in each of these can be further separated by, e.g., treating the exchange field term in the ferromagnetic layer as a perturbation, controlled by a dimensionless perturbation parameter $\lambda_{\mathrm{F}}$. Then the terms with even (odd) powers of $\lambda_{\mathrm{F}}$ in $\chi$ are even (odd) under reversal of the ferromagnetic magnetization $\mathbf{m}$ alone. In practice, since properties of the ferromagnetic layer itself in ST-FMR experiments usually have a very simple dependence on its magnetization direction, we argue that it is likely to be sufficient to stop at the first order in $\lambda_{\mathrm{F}}$. The expressions for the four contributions to $\chi$ obtained by perturbing the equilibrium Liouvillian of the bilayer system under the above approximations are given in the SI, which we apply to a simple model below.

Fig. $2 \mathrm{~h}$ and i show the odd $\left(\tau_{\text {odd }}^{\varphi}\right)$ and even $\left(\tau_{\text {even }}^{\varphi}\right)$ components of $\tau^{\varphi}$ from the experimental data. The odd component under $\mathbf{m}$ reversal is well fit by Eq. (1) from the symmetry analysis, with $b_{2}$, $\mathrm{d}_{5}$, and $\mathrm{d}_{6}$ equal to $-2.0,1.3$, and $-0.7(\mathrm{mT} / \mathrm{A})$, respectively. However, such numbers are still much smaller than the $\tau^{\varphi}$ estimated from the experimental results of $H_{\mathrm{r}}$ shift in Fig. 1, which is about $-83.3 \pm 12.1$ (mT/A). We show below that this discrepancy is caused by the suppression of $\tau^{\varphi}$ by partial cancellation between the STT of the SHE and the FLT of the MSHE. Moreover, $\tau_{\text {even }}^{\varphi}$ is negligibly small compared to $\tau_{\text {odd }}^{\varphi}$, whereas the difference between the $\left|\Delta H_{\mathrm{r}} / I_{\mathrm{dc}}\right|$ in Fig. 1e and $\mathrm{f}$ (of about $10 \%$ ) which is produced by the FLT of the SHE is considerable. This implies that the $\delta s_{z}$ due to the STT of the MSHE and the FLT of the SHE must partially cancel, as discussed further below.

A similar decomposition into odd $\left(\tau_{\text {odd }}^{z}\right)$ and even $\left(\tau_{\text {even }}^{z}\right)$ components of the out-of-plane torque $\tau^{\mathrm{z}}$ from the experimental data is shown in Fig. $2 \mathrm{j}$ and $\mathrm{k}$. Like $\tau^{\varphi}$, the even component of $\tau^{Z}$ is vanishingly small, indicating that the FLT of the MSHE and the STT of the SHE are both small in this $\mathrm{Mn}_{3} \mathrm{Sn} / \mathrm{Ni}-\mathrm{Fe}$ bilayer, or that they partially cancel. In contrast, the $\tau_{\text {odd }}^{z}$ (red circles in Fig. 2j), although much larger, has a dominant contribution from the rf current-induced Oersted field $\tau_{\mathrm{Oe}}^{z}$. The latter is indicated by the black dotted curves in Fig. $2 \mathrm{j}$ and was estimated from measurements in a control sample. Aside from $\tau_{\text {Oe }}^{z}, \tau_{\text {odd }}^{z}$ has contributions from the STT of MSHE and the FLT of SHE. However, from the $H_{\mathrm{r}}$ shift measurement by dc current at $\theta=$ $90^{\circ}$, we found that the contribution of FLT of SHE is very small (Supplementary Fig. 3). Thus, the dominant contribution to $\tau_{\mathrm{odd}}^{z}-\tau_{\mathrm{Oe}}^{z}$ is from the $\delta s_{x, y}$ due to the STT of MSHE.

Modulation of magnetic damping constant by $\tau_{\mathrm{STT}}$. To separate the more conventional SHE-induced STT from $\tau_{\text {odd }}^{\varphi}$, we measured the modulation of magnetic damping constant by dc current. Fig. 3a shows a schematic image of the modulation of magnetic damping torque $\tau_{\mathrm{D}}$ by $\tau_{\mathrm{STT}}$. We set the polar angle $\theta$ to $90^{\circ}$ so that $\tau_{\mathrm{D}}$ is modulated by $\tau_{\text {odd }}^{\varphi}$ of the STT due to the SHE. Fig. $3 \mathrm{~b}, \mathrm{c}$ show experimental results of FMR linewidth $\delta$ at $\varphi=-45^{\circ}$ and $+135^{\circ}$ as a function of applied dc current. We observed linear dependence of $\delta$ on the dc current, with its slope opposite between $\varphi=-45^{\circ}$ and $+135^{\circ}$, consistent with the behavior of the SHE-induced STT. From these data we estimate the spin torque
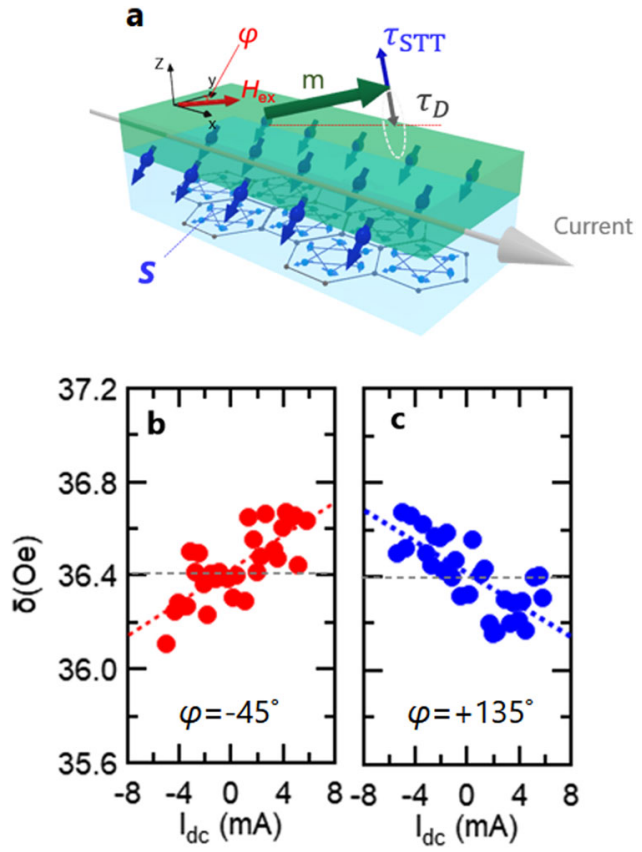

Fig. 3 Modulation of effective magnetic damping by spin Hall effect in $\mathbf{M n}_{\mathbf{3}} \mathbf{S n}$. a Schematic image of modulation of magnetic damping torque $\tau^{\mathrm{D}}$ by STT $\tau_{\mathrm{STT}}$ due to conventional SHE in $\mathrm{Mn}_{3} \mathrm{Sn}$. The polar angle $\theta$ is fixed at $90^{\circ}$. b, c FMR line width $\delta$ at $\varphi=-45^{\circ}(\mathbf{b})$ and $+135^{\circ}$ (c) as a function of applied dc current.

$\tau_{\text {STT }}$ through the formula $\tau_{\text {STT }}=\frac{\gamma \Delta \delta\left(H_{\text {ext }}+M_{\text {eff }} / 2\right)}{2 \pi f \cos \varphi \operatorname{Ic}(M n 3 S n)}$, here $\Delta \delta$ is the dc current-induced change of FMR linewidth. The obtained $\tau_{\text {STT }}$ is $75.0 \pm 6.1(\mathrm{mT} / \mathrm{A})$, which is comparable with the in-plane FLT due to MSHE estimated from the $H_{\mathrm{r}}$ shift in Fig. 1. These experimental results strongly support our hypothesis that the $\tau^{\varphi}$ contributed by the STT component of the SHE suppresses the FLT of MSHE in the same direction in the present system.

Suppression of FLT contribution by inserting the thin $\mathrm{Cu}$ layer. To provide further evidence supporting the hypothesis above, we measured the SOT in a $\mathrm{Mn}_{3} \mathrm{Sn}(140) / \mathrm{Cu}(5) / \mathrm{Ni}-\mathrm{Fe}(15)$ trilayer sample (Fig. 4a). If $\tau^{\varphi}$ due to the FLT of MSHE nearly compensates that due to the STT of SHE as shown in Fig. $2 \mathrm{~h}$, inserting the thin $\mathrm{Cu}$ layer between $\mathrm{Mn}_{3} \mathrm{Sn}$ and Ni-Fe layers should change the angular dependence of the observed $\tau^{\varphi}$ because the FLT is expected to be more strongly suppressed than the STT by a $\mathrm{Cu}$ spacer ${ }^{38}$. Indeed, Fig. $4 \mathrm{~b}$ shows that $\tau_{\text {odd }}^{\varphi}$ in the presence of the spacer has a very different angular dependence from that in Fig. $2 \mathrm{~h}$, and even has a sign change at $\varphi=90^{\circ}$. Moreover, $\tau_{\mathrm{even}}^{\varphi}$ is even enhanced after $\mathrm{Cu}$ insertion compared to Fig. 2i. Both of these changes support our argument that there exists strong compensation between FLTs and STTs along the same directions in $\mathrm{Mn}_{3} \mathrm{Sn} / \mathrm{Ni}-\mathrm{Fe}$ bilayer. This scenario can also be captured by using a toy model mimicking the $\mathrm{Mn}_{3} \mathrm{Sn} / \mathrm{Ni}-\mathrm{Fe}$ interface and applying the quantum kinetic theory results for $\chi$. Qualitatively, one expects $\tau_{\text {odd }}^{\varphi}$ due to either SHE or MSHE to have a dominant $\sin \varphi$ dependence according to Eq. (1), when spin-orbit coupling can be viewed as a perturbation. The unusually $\operatorname{small} \sin \varphi$ component compared to higher-order angular variations suggests accidental cancellation of the leading-order terms due to SHE and MSHE separately.

Comparison spin torque ratio with previous reports. Fig. $5 \mathrm{a}$ shows schematic illustrations of the angular dependence of 

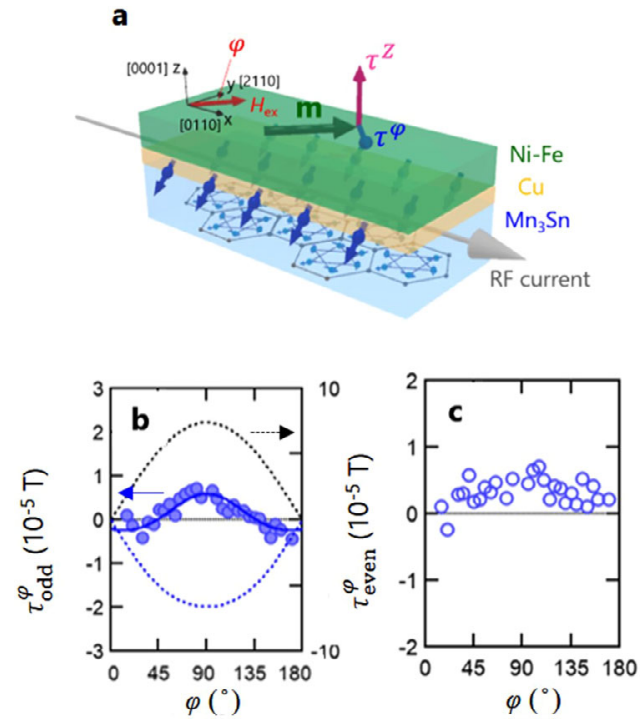

Fig. 4 Spin-torque FMR measurements in $\mathrm{Mn}_{3} \mathrm{Sn} / \mathrm{Cu} / \mathrm{Ni}-\mathrm{Fe}$ trilayer. a Measurement setup for ST-FMR in $\mathrm{Mn}_{3} \mathrm{Sn} / \mathrm{Cu} / \mathrm{Ni}-\mathrm{Fe}$ trilayer. The red and blue arrows on the respective top and bottom surfaces of the $\mathrm{Mn}_{3} \mathrm{Sn}$ stack indicate spin accumulation. The red and blue arrows on the $\mathrm{Ni}-\mathrm{Fe}$ magnetization $\mathbf{m}$ correspond to out-of-plane torque $\tau^{z}$ and in-plane torque $\tau^{\varphi}$. $\mathbf{b}, \mathbf{c}$ odd-(b) and even-(c) components of $\tau^{\varphi}$ as a function of the in-plane m direction $\varphi$. Blue (Black) dashed line corresponds to $\tau^{\varphi}$ due to the STT of the conventional SHE (FLT of the MSHE).
$\tau_{\mathrm{STT}}$ and $\tau_{\mathrm{FLT}}$ in a $\mathrm{Mn}_{3} \mathrm{Sn} / \mathrm{Ni}-\mathrm{Fe}$ bilayer, obtained by taking all the experimental data and analyses above into account. The out-of-plane (in-plane) component of $\tau_{\text {STT }}$ is mainly due to the MSHE (SHE), while the $\tau_{\mathrm{FLT}}$ has a dominant in-plane component mostly contributed by the MSHE. For a qualitative comparison with the SOT in conventional bilayer systems, we also plotted in Fig. 5a spin accumulation $\mathbf{S}$ under the assumption that $\tau_{\text {STT }}$ can be understood as due to the diffusion of $\mathbf{S}$ into the FM layer. Such a representation shows that the polarization direction of the spin accumulation at the $\mathrm{Mn}_{3} \mathrm{Sn}$ surface is tilted out of the $x y$ plane.

Finally, a quantitative comparison is made between the spin torque ratio $\theta$ in the present system and those in previous reports (Fig. 5b) by means of the same manner with $\mathrm{SHE}^{30}$. $\theta_{\mathrm{FLT}(\mathrm{STT})}=\frac{\left(\frac{2 e}{h}\right) \sigma_{\mathrm{S}}^{\mathrm{ELT}(\mathrm{STT})}}{\sigma_{\mathrm{C}}}=\frac{\left(\frac{2 e}{\hbar}\right) \tau_{\mathrm{FLT}(\mathrm{STT})} M_{\mathrm{S}} t_{\mathrm{FM}}}{S}$, where $\sigma_{\mathrm{C}(\mathrm{S})}$ and $S$ are the charge conductivity, spin current conductivity in $\mathrm{Mn}_{3} \mathrm{Sn}$ and cross-sectional area onf $\mathrm{Mn}_{3} \mathrm{Sn}$ layer. The $\theta$, in particular the FLT due to the MSHE, are more substantial than the reported values in paramagnetic metals such as $\mathrm{Pt}^{30,39,40}, \quad \beta-\mathrm{Ta}^{8,41}, \quad \beta-\mathrm{W}^{9,42}$. Estimated spin current conductivity $\sigma_{\mathrm{S}}^{\mathrm{FLT}}$ due to MSHE is $2.8 \pm 0.4 \times 10^{5}\left(\hbar / 2 \mathrm{e} \Omega^{-1} \mathrm{~m}^{-1}\right)$, which is comparable with the calculated values in chiral antiferromagnets ${ }^{40,43,44}$. We note much larger FLT comes from the MSHE than from the SHE in $\mathrm{Mn}_{3} \mathrm{Sn}$. This is because the former has an inter-band nature and could benefit from the strong momentum-dependent spin splitting in $\mathrm{Mn}_{3} \mathrm{Sn}$. Giant FLTs due to the SHE have been observed in topological insulator/FM ${ }^{45,46}$ and $\mathrm{TMD}$ material/FM

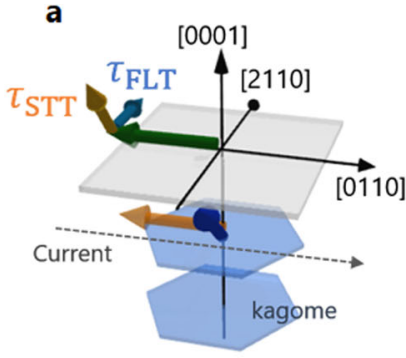

$\varphi=-90^{\circ}$

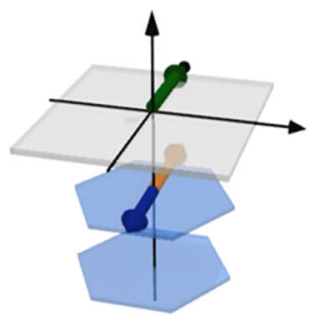

$\varphi=0^{\circ}$

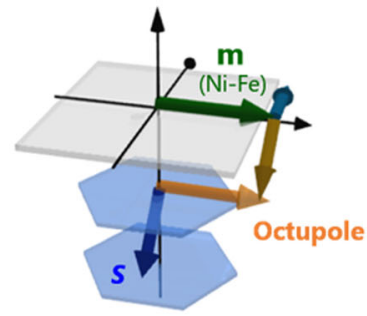

$\varphi=+90^{\circ}$

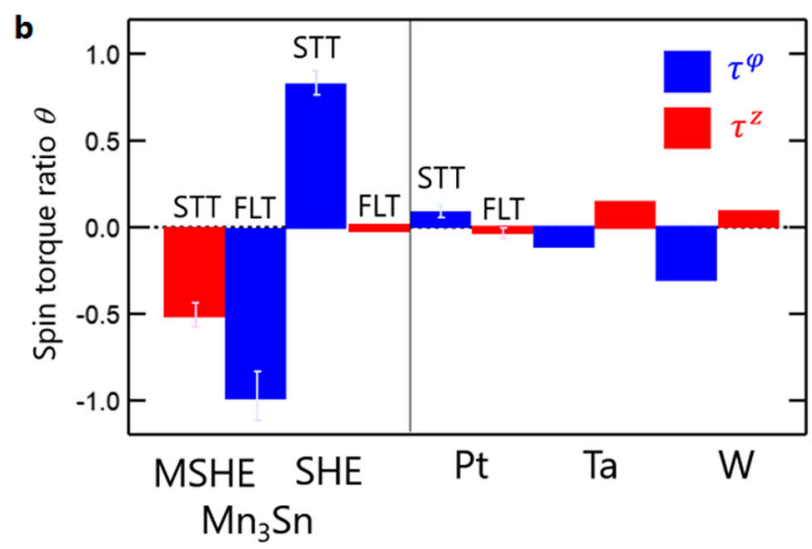

Fig. $\mathbf{5}$ Giant field-like torque and spin polarization vector of spin accumulation in $\mathbf{M n}_{\mathbf{3}} \mathbf{S n} \mathbf{N i}$-Fe bilayer. a Schematic images of spin polarization vector $\mathbf{S}$ (blue arrow) of spin accumulation at $\mathrm{Mn}_{3} \mathrm{Sn} / \mathrm{Ni}$-Fe interface and spin transfer torque (STT) and field-like torque (FLT) as a function of the in-plane direction $\varphi$ of the magnetization $\mathbf{m}$ (green arrow) in Ni-Fe layer. $\mathbf{b}$ Comparisons of spin torque ratio $\theta$ in $\mathrm{Mn}_{3} \mathrm{Sn}$ and that in typical transition metals. Blue and red bars correspond to $\tau^{\varphi}$ and $\tau^{2}$ at $\varphi=90^{\circ}$. The sign of spin torque due to MSHE is changed for $\varphi=-90^{\circ}$. 
heterostructures ${ }^{20,47,48}$, which usually involve quasi-2D systems with large spin-orbit coupling at the interfaces. In near future, by utilizing the high-quality $\mathrm{Mn}_{3} X$ thin film/ferromagnetic heterostructure, the giant FLT, as well as the STT due to bulk $\mathrm{Mn}_{3} \mathrm{Sn}$, should directly contribute to realizing field-free magnetization switching ${ }^{49}$, high-speed magnetization control $^{7}$, and domain wall manipulation ${ }^{50}$. Our findings thus provide a route for efficient manipulation of magnetic states and realization of novel functionalities by utilizing topological Weyl antiferromagnets.

\section{Methods}

Sample fabrication. The single crystal $\mathrm{Mn}_{3} \mathrm{Sn}$ used in the present study was prepared by using the Bridgman technique. The rectangular element, cut out of the single crystal using the Focused Ion Beam, transferred to the co-planar waveguide of 5-nm Ti /200-nm Au on a thermally oxidized Si substrate by using a W needle manipulator. The surface of the $\mathrm{Mn}_{3} \mathrm{Sn}$ element was cleaned by Ar-ion milling to remove the residual damaged layer. The $\mathrm{NiFe}$ and $\mathrm{Al}_{2} \mathrm{O}_{3}$ layer was formed by optical photolithography and lift-off process. The resistivity of the processed $\mathrm{Mn}_{3} \mathrm{Sn}$ crystal was $360 \mu \Omega \mathrm{cm}$ at room temperature, which is comparable to the bulk sample ${ }^{14}$

ST-FMR measurement set up. An rf current is applied along the long edge of the rectangle by a microwave analog signal generator (Keysight: MXG N5183A). An external static magnetic field $H_{\text {ext }}$ in the range from 0 to $4.0 \mathrm{kOe}$ is also applied. All the experiments are performed at room temperature. For resonance field shift measurement in Fig. 1, we used the dc current source (Yokogawa GS200) and lock in amplifier (NF LI5640).

\section{Data availability}

The datasets generated and analyzed during this study are available from the corresponding authors on reasonable request.

\section{Code availability}

The corresponding computer codes are available from the corresponding authors on reasonable request.

Received: 23 May 2021; Accepted: 27 September 2021; Published online: 18 November 2021

\section{References}

1. Dyakonov, M. I. \& Perel, V. I. Possibility of orientating electron spins with current. Sov. J. Exp. Theor. Phys. Lett. 13, 467-469 (1971).

2. Hirsch, J. E. Spin Hall effect. Phys. Rev. Lett. 83, 1834-1837 (1999).

3. Kato, Y. K., Myers, R. C., Gossard, A. C. \& Awschalom, D. D. Observation of the spin Hall effect in semiconductors. Science 306, 1910-1913 (2004).

4. Wunderlich, J., Kaestner, B., Sinova, J. \& Jungwirth, T. Experimental observation of the spin-Hall effect in a two-dimensional spin-orbit coupled semiconductor system. Phys. Rev. Lett. 94, 047204 (2005).

5. Kimura, T., Otani, Y., Sato, T., Takahashi, S. \& Maekawa, S. Roomtemperature reversible spin Hall effect. Phys. Rev. Lett. 98, 156601 (2007).

6. Sinova, J., Valenzuela, S. O., Wunderlich, J., Back, C. H. \& Jungwirth, T. Spin Hall effects. Rev. Mod. Phys. 87, 1213-1260 (2015).

7. Legrand, W., Ramaswamy, R., Mishra, R. \& Yang, H. Coherent subnanosecond switching of perpendicular magnetization by the fieldlike spin-orbit torque without an external magnetic field. Phys. Rev. Appl. 3, https://doi.org/10.1103/PhysRevApplied.3.064012 (2015).

8. Liu, L. et al. Spin-torque switching with the giant spin Hall effect of tantalum. Science 336, 555-558 (2012).

9. Pai, C.-F. et al. Spin transfer torque devices utilizing the giant spin Hall effect of tungsten. Appl. Phys. Lett. 101, https://doi.org/10.1063/1.4753947 (2012).

10. Cubukcu, M. et al. Spin-orbit torque magnetization switching of a threeterminal perpendicular magnetic tunnel junction. Appl. Phys. Lett. 104, https://doi.org/10.1063/1.4863407 (2014)

11. Cai, K. et al. Electric field control of deterministic current-induced magnetization switching in a hybrid ferromagnetic/ferroelectric structure. Nat. Mater. 16, 712-716 (2017).

12. Fukami, S., Zhang, C., DuttaGupta, S., Kurenkov, A. \& Ohno, H. Magnetization switching by spin-orbit torque in an antiferromagnetferromagnet bilayer system. Nat. Mater. 15, 535-541 (2016).

13. $\mathrm{Yu}, \mathrm{G}$. et al. Switching of perpendicular magnetization by spin-orbit torques in the absence of external magnetic fields. Nat. Nanotechnol. 9, 548-554 (2014).
14. Wang, W. et al. Anomalous spin-orbit torques in magnetic single-layer films. Nat. Nanotechnol. 14, 819-824 (2019).

15. Iihama, S. et al. Spin-transfer torque induced by the spin anomalous Hall effect. Nat. Electron. 1, 120-123 (2018).

16. Nan, T. et al. Controlling spin current polarization through non-collinear antiferromagnetism. Nat. Commun. 11, 4671 (2020).

17. Chen, X. et al. Observation of the antiferromagnetic spin Hall effect. Nat. Mater. https://doi.org/10.1038/s41563-021-00946-z (2021).

18. Baek, S. C. et al. Spin currents and spin-orbit torques in ferromagnetic trilayers. Nat. Mater. 17, 509-513 (2018).

19. MacNeill, D. et al. Control of spin-orbit torques through crystal symmetry in WTe2/ferromagnet bilayers. Nat. Phys. 13, 300-305 (2016).

20. Guimaraes, M. H. D., Stiehl, G. M., MacNeill, D., Reynolds, N. D. \& Ralph, D. C. Spin-orbit torques in NbSe2/permalloy bilayers. Nano Lett. 18, 1311-1316 (2018).

21. Nakatsuji, S., Kiyohara, N. \& Higo, T. Large anomalous Hall effect in a noncollinear antiferromagnet at room temperature. Nature 527, 212-215 (2015).

22. Ikhlas, M. et al. Large anomalous Nernst effect at room temperature in a chiral antiferromagnet. Nat. Phys. 13, 1085-1090 (2017).

23. Higo, T. et al. Large magneto-optical Kerr effect and imaging of magnetic octupole domains in an antiferromagnetic metal. Nat. Photonics 12, 73-78 (2018).

24. Higo, T. et al. Omnidirectional control of large electrical output in a topological antiferromagnet. Adv. Funct. Mater. 31, 2008971 (2021).

25. Kuroda, K. et al. Evidence for magnetic Weyl fermions in a correlated metal. Nat. Mater. 16, 1090-1095 (2017).

26. Chen, T. et al. Anomalous transport due to Weyl fermions in the chiral antiferromagnets Mn3X, X = Sn, Ge. Nat. Commun. 12, 572 (2021).

27. Tsai, H. et al. Electrical manipulation of a topological antiferromagnetic state. Nature 580, 608-613 (2020).

28. Kimata, M. et al. Magnetic and magnetic inverse spin Hall effects in a noncollinear antiferromagnet. Nature 565, 627-630 (2019).

29. Manchon, A. et al. Current-induced spin-orbit torques in ferromagnetic and antiferromagnetic systems. Rev. Modern Phys. 91, https://doi.org/10.1103/ RevModPhys.91.035004 (2019).

30. Liu, L., Moriyama, T., Ralph, D. C. \& Buhrman, R. A. Spin-torque ferromagnetic resonance induced by the spin Hall effect. Phys. Rev. Lett. 106, 036601 (2011).

31. Mellnik, A. R. et al. Spin-transfer torque generated by a topological insulator Nature 511, 449-451 (2014).

32. Kondou, K. et al. Fermi-level-dependent charge-to-spin current conversion by Dirac surface states of topological insulators. Nat. Phys. 12, 1027-1031 (2016).

33. Kubota, H. et al. Quantitative measurement of voltage dependence of spintransfer torque in MgO-based magnetic tunnel junctions. Nat. Phys. 4, 37-41 (2007).

34. Sankey, J. C. et al. Measurement of the spin-transfer-torque vector in magnetic tunnel junctions. Nat. Phys. 4, 67-71 (2007).

35. Freimuth, F., Blügel, S. \& Mokrousov, Y. Spin-orbit torques in $\mathrm{Co} / \mathrm{Pt}(111)$ and Mn/W(001) magnetic bilayers from first principles. Phys. Rev. B 90, https:// doi.org/10.1103/PhysRevB.90.174423 (2014).

36. Hals, K. M. D. \& Brataas, A. Spin-motive forces and current-induced torques in ferromagnets. Phys. Rev. B 91, https://doi.org/10.1103/PhysRevB.91.214401 (2015).

37. Freimuth, F., Blügel, S. \& Mokrousov, Y. Direct and inverse spin-orbit torques Phys. Rev. B 92, https://doi.org/10.1103/PhysRevB.92.064415 (2015).

38. Bonell, F. et al. Control of spin-orbit torques by interface engineering in topological insulator heterostructures. Nano Lett. 20, 5893-5899 (2020).

39. Kondou, K., Sukegawa, H., Mitani, S., Tsukagoshi, K. \& Kasai, S. Evaluation of spin Hall angle and spin diffusion length by using spin current-induced ferromagnetic resonance. Appl. Phys. Exp. 5, https://doi.org/10.1143/ apex.5.073002 (2012).

40. Pai, C.-F., Ou, Y., Vilela-Leão, L. H., Ralph, D. C. \& Buhrman, R. A Dependence of the efficiency of spin Hall torque on the transparency of $\mathrm{Pt} /$ ferromagnetic layer interfaces. Phys. Rev. B 92, https://doi.org/10.1103/ PhysRevB.92.064426 (2015).

41. Cecot, M. et al. Influence of intermixing at the $\mathrm{Ta} / \mathrm{CoFeB}$ interface on spin Hall angle in $\mathrm{Ta} / \mathrm{CoFeB} / \mathrm{MgO}$ heterostructures. Sci. Rep. 7, 968, https:// doi.org/10.1038/s41598-017-00994-z (2017).

42. Kim, C., Kim, D., Chun, B. S., Moon, K.-W. \& Hwang, C. Evaluation method for fieldlike-torque efficiency by modulation of the resonance field. Phys. Rev. Appl. 9, https://doi.org/10.1103/PhysRevApplied.9.054035 (2018).

43. Zhang, Y. et al. Strong anisotropic anomalous Hall effect and spin Hall effect in the chiral antiferromagnetic compoundsMn3X $(\mathrm{X}=\mathrm{Ge}, \mathrm{Sn}, \mathrm{Ga}, \mathrm{Ir}, \mathrm{Rh}$, and $\mathrm{Pt})$. Phys. Rev. B 95, https://doi.org/10.1103/PhysRevB.95.075128 (2017).

44. Zhang, Y., Železný, J., Sun, Y., van den Brink, J. \& Yan, B. Spin Hall effect emerging from a noncollinear magnetic lattice without spin-orbit coupling. $N$. J. Phys. 20, https://doi.org/10.1088/1367-2630/aad1eb (2018). 
45. Nayak, A. K. et al. Large anomalous Hall effect driven by a nonvanishing Berry curvature in the noncolinear antiferromagnet Mn3Ge. Sci. Adv. 2, e1501870 (2016)

46. Wang, Y. et al. Topological surface states originated spin-orbit torques in $\mathrm{Bi}(2)$ Se(3). Phys. Rev. Lett. 114, 257202 (2015).

47. Chi, Z. et al. The spin Hall effect of Bi-Sb alloys driven by thermally excited Dirac-like electrons. Sci. Adv. 6, eaay2324 (2020).

48. Shao, Q. et al. Strong Rashba-Edelstein effect-induced spin-orbit torques in monolayer transition metal dichalcogenide/ferromagnet bilayers. Nano Lett. 16, 7514-7520 (2016).

49. Lee, O. J. et al. Central role of domain wall depinning for perpendicular magnetization switching driven by spin torque from the spin Hall effect. Phys. Rev. B 89, https://doi.org/10.1103/PhysRevB.89.024418 (2014).

50. Manipatruni, S., Nikonov, D. E. \& Young, I. A. Energy-delay performance of giant spin Hall effect switching for dense magnetic memory. Appl. Phys. Exp. 7, https://doi.org/10.7567/apex.7.103001 (2014)

\section{Acknowledgements}

This work was supported by JST-CREST(No. JPMJCR18T3) and JST-Mirai(JPMJMI20A1) and by Grants-in-Aid for Scientific Research (19H05629, 19H02586, 19H00650). H.C. was supported by the National Science Foundation CAREER Grant DMR-1945023. A.H.M. was supported by the Army Research Office under Grant Number W911NF-16-1-0472. Institute for Quantum Matter, an Energy Frontier Research Center was funded by DOE, Office of Science, Basic Energy Sciences under Award DE-SC0019331.

\section{Author contributions}

Y.O. and S.N. planned the experimental project. K.K., S.N. and Y.O. planned the experiments. K.K. performed the measurements and data analyses. T.T., M.I. and T.H. contributed to the crystal growth and device fabrication. H.C. and A.H.M. performed the theoretical analyses. K.K., H.C. A.H.M., S.N. and Y.O wrote the manuscript. All authors discussed the results and commented on the manuscript.

\section{Competing interests}

The authors declare no competing interests.

\section{Additional information}

Supplementary information The online version contains supplementary material available at https://doi.org/10.1038/s41467-021-26453-y.

Correspondence and requests for materials should be addressed to Kouta Kondou or YoshiChika Otani.

Peer review information Nature Communications thanks Cheng Song and the other, anonymous, reviewer(s) for their contribution to the peer review of this work.

Reprints and permission information is available at http://www.nature.com/reprints

Publisher's note Springer Nature remains neutral with regard to jurisdictional claims in published maps and institutional affiliations.

\section{(c) (i)}

Open Access This article is licensed under a Creative Commons Attribution 4.0 International License, which permits use, sharing, adaptation, distribution and reproduction in any medium or format, as long as you give appropriate credit to the original author(s) and the source, provide a link to the Creative Commons license, and indicate if changes were made. The images or other third party material in this article are included in the article's Creative Commons license, unless indicated otherwise in a credit line to the material. If material is not included in the article's Creative Commons license and your intended use is not permitted by statutory regulation or exceeds the permitted use, you will need to obtain permission directly from the copyright holder. To view a copy of this license, visit http://creativecommons.org/ licenses/by/4.0/.

(C) The Author(s) 2021 